\title{
Transition Probabilities at Crossing in the Landau-Zener Problem
}

\author{
Tae Jun Park \\ Department of Chemistry, Dongguk University, Seoul 100-715, Korea. "E-mail: tjpark@dgu.edu \\ Received July 18, 2005
}

\begin{abstract}
We obtain probabilities at a crossing of two linearly time-dependent potentials that are constantly coupled to the other by solving a time-dependent Schrödinger equation. We find that the system which was initially localized at one state evolves to split into both states at the crossing. The probability splitting depends on the coupling strength $V_{0}$ such that the system stays at the initial state in its entirety when $V_{0}=0$ while it is divided equally in both states when $V_{0} \rightarrow \infty$. For a finite coupling the probability branching at the crossing is not even and thus a complete probability transfer at $t \rightarrow \infty$ is not achieved in the linear potential crossing problem. The Landau-Zener formula for transition probability at $t \rightarrow \infty$ is expressed in terms of the probabilities at the crossing.
\end{abstract}

Key Words : Nonadiabatic transition, Landau-Zener, Time-dependent, Crossing

\section{Introduction}

Nonadiabatic transitions, as being implied, take place between adiabatic states when the adiabaticity of dynamics does not hold due to rapid change of adiabatic parameters. One typical example is the breakdown of Born-Oppenheimer approximation in molecules with fast nuclear motions where the electronic transitions are induced. These transitions often occur in regions of avoided crossings at which the difference of energies between states are small and/or the couplings between states are strong. Hence nonadiabatic transitions must be taken into account in reactions with high energies and for molecules under electromagnetic fields where the level crossings are introduced by the field. ${ }^{1}$

Many types of potential curve crossings are used to investigate nonadiabatic transitions theoretically in diabatic representations. Curve crossings in a coordinate space have been mainly considered to account for the transitions. Crossing problems in time domain draw rising attention as the interest for controlling molecular processes with lasers become increased..$^{2-8}$ Radiative transitions, for instance, are treated as nonadiabatic transitions by regarding them as curve crossings between the Floquet (dressed) states. ${ }^{9,10}$ The simplest crossing system is probably the crossing of two linear potential curves that are constantly coupled to each other. This is the well known Landau-Zener problem. ${ }^{11}$ Zener solved it analytically and determined the transition probability assuming the particle velocity to be constant ${ }^{12}$. Studies on other crossing problems have followed the pioneering work and such problems of non-curve crossings ${ }^{13}$ and the exponential potential crossings ${ }^{14,15}$ have been solved quantum mechanically. Exact solutions for the linear curve crossing, though not analytic in form, have also been obtained without constant velocity assumption. ${ }^{16,17}$ Meanwhile solutions for general two state curve crossings have been derived semiclassically using Wentzel-Kramers-Brilloin (WKB) theory in association with Stokes phenomena. ${ }^{18-20}$
Owing to the continuing effort of many researchers, the significance of nonadiabatic transitions is realized in various processes of physics and chemistry. ${ }^{1}$

The Landau-Zener formula ${ }^{12}$ is a high energy approximation of the potential crossing where two diabatic potentials are linear in coordinates and their coupling is constant. ${ }^{21}$ It represents the transition probabilities evaluated at $t \rightarrow \infty$. However, the formula is the exact solution for the crossing of linear potentials in time. ${ }^{1}$ It is interesting and useful to determine the transition probabilities at the crossing point $(t=0)$ of the Landau-Zener model since these probabilities for one passage not only reproduce the transition probabilities of the model at $t \rightarrow \infty$ but can be used to evaluate the transition matrix for controlling the system with linearly chirped fields. ${ }^{7}$ Furthermore they may be used to calculate transition probabilities for the crossing of potentials which are locally approximated to be linear.

In this work, the transition probability at crossing is analytically obtained for the time-dependent Landau-Zener model. Final transition probability, namely the probability at $t \rightarrow \infty$ (the Landau-Zener formula) is expressed in terms of the probability at the crossing.

\section{Time-Dependent Landau-Zener Problem}

Consider following coupled time-dependent Schrödinger equations;

$$
\begin{aligned}
& i \hbar \frac{d c_{1}(t)}{d t}=V_{1}(t) c_{1}(t)+V_{0} c_{2}(t) \\
& i \hbar \frac{d c_{2}(t)}{d t}=V_{0} c_{1}(t)+V_{2}(t) c_{2}(t)
\end{aligned}
$$

where two diabatic potentials $V_{1}(t)$ and $V_{2}(t)$ are linear functions of time and $V_{0}$ is constant. It is assumed that the slope of $V_{1}(t)$ is greater than the slope of $V_{2}(t)$ and that two functions cross at $t=0$. The model described by these equations represents the Floquet Hamiltonian of the two- 
level system with energies $E_{1}$ and $E_{2}$ under the linearly chirped constant intensity laser field of $E(t)=\varepsilon \cos \int w(t) d t$ where the frequency $w(t)$ is linear in time and the pulse envelope $\varepsilon$ is constant. ${ }^{7}$ The conditions for solving above equations are chosen so that initially the system is under $V_{1}(t)$ and they are given as follows;

$$
\begin{gathered}
\left|c_{1}(-\infty)\right|=1 \\
c_{2}(-\infty)=0
\end{gathered}
$$

Let us introduce $U(t)$ and $\gamma$ which are defined

$$
U(t)=e^{\frac{i}{2 \hbar} \int V_{1}+V_{2} d t} c_{2}(t)
$$

and

$$
\gamma=\frac{d V_{1}}{d t}-\frac{d V_{2}}{d t}
$$

where $\gamma$ is a positive constant since the slope of $V_{1}(t)$ is larger than that of $V_{2}(t)$.

The second-order time derivative of $c_{2}(t)$ leads to the following equation for $U(t)$

$$
\frac{d^{2} U}{d t^{2}}+\left[\frac{1}{4}\left(\frac{\gamma}{\hbar}\right)^{2} t^{2}-i \frac{\gamma}{2 \hbar}+\frac{V_{0}^{2}}{\hbar^{2}}\right] U=0
$$

and a transformation of variable $t$ as below

$$
z=\sqrt{\frac{\gamma}{\hbar}} e^{-i \frac{\pi}{4}} t
$$

reduces Eq. (5) to the Weber equation

$$
\frac{d^{2} U}{d t^{2}}+\left[\frac{1}{2}+i \Delta-\frac{z^{2}}{4}\right] U=0
$$

with $\Delta=V_{0}^{2} / \hbar \gamma$. The parabolic cylinder function $D_{-i \Delta-1}(\mp i z)^{22}$ is a particular solution of Eq. (7) depending on the sign of $\Delta(-$ for $\Delta>0$ and + for $\Delta<0)$. Since $\Delta$ is assumed positive, the solution is chosen as

$$
U(z)=A_{-} D_{-i \Delta-1}(-i z)
$$

which vanishes as $t \rightarrow-\infty$ to satisfy Eq. (2b). This is realized from that $D_{n}(y)$ vanishes when $|\arg (y)|<3 / 4 \pi$ for infinite $y$ by $D_{n}(y) \sim y^{n} \exp \left(-1 / 4 y^{2}\right)^{23}$ and from that $\arg (-i z)=\pi / 4$ is in the corresponding sector as $t \rightarrow-\infty$. The constant $A_{-}$will be determined later. We obtain $c_{2}(t)$ by placing $U(t)$ in Eq. (3) and then find $c_{1}(t)$ in Eq. (1b). They are given as follows;

$$
\begin{gathered}
c_{1}(t)=\frac{A_{-}}{V_{0}}\left[\frac{V_{1}-V_{2}}{2} D_{-i \Delta-1}+\sqrt{\gamma \hbar} e^{\frac{\pi}{4} i} \frac{d D_{-i \Delta-1}}{d z}\right] e^{-\frac{i}{2 \hbar} \int V_{1}+V_{2} d t} \\
c_{2}(t)=A_{-} D_{-i \Delta-1} e^{-\frac{i}{2 h} \int V_{1}+V_{2} d t}
\end{gathered}
$$

Since $V_{1}(t)$ and $V_{2}(t)$ cross at $t=0, c_{1}(0)$ and $c_{2}(0)$ are calculated as follows;

$$
\begin{aligned}
& c_{1}(0)=\frac{A_{-}}{V_{0}} \sqrt{\gamma \hbar} e^{\frac{\pi}{4} i} D_{-i \Delta-1}^{\prime}(0) \\
& c_{2}(0)=A_{-} D_{-i \Delta-1}(0)
\end{aligned}
$$

where $D_{-i \Delta-1}^{\prime}(0)=\left.\frac{d D_{-i \Delta-1}(-i z)}{d z}\right|_{z=0}$. Values of $D_{-i \Delta-1}(0)$ and $D_{-i \Delta-1}^{\prime}(0)$ are explicitly known ${ }^{22}$ as below

$$
\begin{gathered}
D_{-i \Delta-1}(0)=-i \sqrt{2} \frac{\Gamma(-i \Delta)}{\Gamma\left(\frac{1}{2}-i \frac{\Delta}{2}\right)} 2^{i \frac{\Delta}{2}} \sinh \frac{\pi \Delta}{2} \\
D_{-i \Delta-1}^{\prime}(0)=2 i \frac{\Gamma(-i \Delta)}{\Gamma\left(-i \frac{\Delta}{2}\right)} 2^{i \frac{\Delta}{2}} \cosh \frac{\pi \Delta}{2}
\end{gathered}
$$

where $\Gamma(z)$ is the gamma function. Substitution of these expressions in Eqs. (10a) and (10b) determines $\left|c_{1}(0)\right|^{2}$ and $\left|c_{2}(0)\right|^{2}$ as

$$
\begin{aligned}
& \left|c_{1}(0)\right|^{2}=\frac{1}{\Delta}\left|A_{-}\right|^{2} \cosh \frac{\pi}{2} \Delta \\
& \left|c_{2}(0)\right|^{2}=\frac{1}{\Delta}\left|A_{-}\right|^{2} \sinh \frac{\pi}{2} \Delta
\end{aligned}
$$

Since the sum of $\left|c_{1}(0)\right|^{2}$ and $\left|c_{2}(0)\right|^{2}$ is equal to 1 from the initial condition (Eqs. (2a) and (2b)), $\left|A_{-}\right|$is to be

$$
\left|A_{-}\right|=\sqrt{\Delta} \exp \left(-\frac{\pi}{4} \Delta\right)
$$

The probabilities at crossing are then obtained as follows;

$$
\begin{aligned}
& P_{1}(0)=\frac{1}{2}\left(1+e^{-\pi \Delta}\right) \\
& P_{2}(0)=\frac{1}{2}\left(1-e^{-\pi \Delta}\right)
\end{aligned}
$$

where $P_{1}(0)$ and $P_{2}(0)$ represent probabilities for the system to be in state 1 and in state 2 at $t=0$ respectively. It shows that the probability splits due to the potential crossing although the system was entirely in the state 1 initially $\left(P_{1}(-\infty)=1\right)$. Unless the coupling is infinitely large $(\Delta \rightarrow \infty), P_{1}(0)$ is not equal to $P_{2}(0)$. The complete probability transfer at $t \rightarrow \infty$ is not achieved in the LandauZener model since the probabilities at crossings can not be made $1 / 2$ by varying parameters.

The probability $P_{1}(\infty)$ is equal to $\left|c_{1}(\infty)\right|^{2}$ and it is calculated using Eq. (9a) and the asymptotic formula of $D_{-i \Delta}(-i z)$. First the following recurrence relation ${ }^{22}$ for $D_{-i \Delta-1}^{\prime}(-i z)$ is inserted in Eq. (9a);

$$
D_{-i \Delta-1}^{\prime}(-i z)=i\left(\frac{1}{2}(-i z) D_{-i \Delta-1}(-i z)-D_{-i \Delta}(-i z)\right)
$$

where $D_{-i \Delta-1}^{\prime}(-i z)=d D_{-i \Delta-1}(-i z) / d z$. When Eq. (6) is used, $c_{1}(t)$ is obtained as follows; 


$$
c_{1}(t)=-\frac{A_{-}}{V_{0}} \sqrt{\gamma \hbar} e^{-\frac{\pi}{4} i} D_{-i \Delta}(-i z)
$$

As $t \rightarrow \infty$ (thus $-i z \sim e^{-\frac{3}{4} \pi i}{ }^{\infty}$ ), the asymptotic formula for $D_{-i \Delta}(-i z)$ at $\arg (-i z)=-\frac{3}{4} \pi$ is given as below ${ }^{23}$;

$$
\begin{array}{r}
D_{-i \Delta}\left(e^{-\frac{3}{4} \pi i} \infty\right) \sim{ }_{R \rightarrow \infty}\left(e^{-\frac{3}{4} \pi i} R\right)^{-i \Delta} e^{\frac{i}{4} R^{2}} \\
-\frac{\sqrt{2 \pi}}{\Gamma(i \Delta)} e^{-\pi \Delta}\left(e^{-\frac{3}{4} \pi i} R\right)^{i \Delta-1} e^{-\frac{i}{4} R^{2}}
\end{array}
$$

in which the second term is to vanish due to the factor $R^{-1}$ as $R \rightarrow \infty$. The value $\left|c_{1}(\infty)\right|^{2}$ is then to be

$$
\left|c_{1}(\infty)\right|^{2}=\left|\frac{A_{-}}{V_{0}}\right|^{2} \gamma \hbar \exp \left(-\frac{3}{2} \pi \Delta\right)=\exp (-2 \pi \Delta)
$$

where Eq. (13) is used for $\left|A_{-}\right|$. This is just the probability $P_{1}(\infty)$. The famous Landau-Zener probability which is given by Zener ${ }^{12}$ exactly coincides with Eq. (18) and it represents the probability that the system stays in the state 1 at $t \rightarrow \infty$. The probability for transition from state 1 to state 2, $P_{2}(\infty)$ can be expressed in terms of the probability at $t=0$ as;

$$
P_{2}(\infty)=4 P_{1}(0)\left(1-P_{1}(0)\right)
$$

which is similar to the Zhu-Nakamura formula for the linear curve crossing in coordinate space. ${ }^{24}$ The result signifies that the final probabilities are determined by the probabilities at crossing which could be useful to apply in control problems.

\section{Conclusion}

Probabilities at a crossing of two linearly time-dependent potentials with a constant coupling are analytically obtained by solving a time-dependent coupled Schrödinger equation which is the time-dependent Landau-Zener problem. The system which was initially localized in state 1 evolves to split into both states at the crossing. The probability splitting depends on the coupling strength $V_{0}$ such that the system stays at the initial state in its entirety when $V_{0}=0$ while it splits equally at both states when $V_{0} \rightarrow \infty$. For a finite coupling the probability branching is not even and thus a complete probability transfer at $t \rightarrow \infty$ is not achieved in the crossing of two linear potentials. The Landau-Zener formula for transition probability at $t \rightarrow \infty$ is calculated in terms of the probabilities at the crossing. Considering a radiative transition by a linearly chirped field is equivalent to the crossing of two linear potentials, it is further realized that a complete control of the corresponding transition may not be feasible by a sequence of linearly chirped pulses.

Acknowledgment. This work was supported by the research program of Dongguk University.

\section{References}

1. Nakamura, H. Nonadiabatic Transition: Concpets, Basic Theories, and Applications; World Scientific: Singapore, 2002.

2. Shore, B. W.; Bergmann, K.; Kuhn, A.; Schiemann, S.; Oreg, J. Phys. Rev. A 1992, 45, 5297.

3. Teranishi, Y.; Nakamura, H. Phys. Rev. Lett. 1998, 81, 2032.

4. Teranishi, Y.; Nakamura, H. J. Chem. Phys. 1999, 111, 1415.

5. Teranishi, Y.; Nagaya, K.; Nakamura, H. In Advances in Multiphoton Processes and Spectroscopy; Gordon, R. J.; Fujimura, Y., Eds.; World Scientific: Singapore, 2001; Vol. 14.

6. Nagaya, K.; Teranishi, Y.; Nakamura, H. In Laser Control and Manipulation of Molecules; Bandrauk, A. D.; Gordon, R. J.; Fujimura, Y., Eds.; American Chemical Society: Washington, DC, 2002.

7. Nagaya, K.; Teranishi, Y.; Nakamura, H. J. Chem. Phys. 2002, $117,9588$.

8. Zou, S.; Kondorskiy, A.; Mil'nikov, G.; Nakamura, H. J. Chem. Phys. 2005, 122, 084112 .

9. Shirley, J. H. Phys. Rev. 1965, 138, B979.

10. Ho, T.-S.; Chu, S.-I. Chem. Phys. Lett. 1987, 141, 315.

11. Landau, L. D. Phys. Zts. Sov. 1932, 2, 46.

12. Zener, C. Proc. Roy. Soc. 1932, A137, 696.

13. Rosen, N.; Zener, C. Phys. Rev. 1932, 40, 502.

14. Osherov, V. I.; Nakamura, H. J. Chem. Phys. 1996, 105, 2770.

15. Osherov, V. I.; Nakamura, H. Phys. Rev. 1998, A59, 2486.

16. Zhu, C.; Nakamura, H. J. Chem. Phys. 1992, 97, 1892.

17. Zhu, C.; Nakamura, H. J. Chem. Phys. 1992, 97, 8497.

18. Zhu, C.; Nakamura, H. J. Chem. Phys. 1994, 101, 10630.

19. Zhu, C.; Nakamura, H. J. Chem. Phys. 1995, 102, 7448.

20. Zhu, C.; Nakamura, H. J. Chem. Phys. 1998, 109, 4689.

21. Nakamura, H. Chem. Phys. 2003, 295, 269.

22. Abramowitz, M.; Stegun, I. A. Handbook of Mathematical Functions; Dover: New York, 1970; p 685.

23. Child, M. S. Molecular Collision Theory; Academic Press: London, 1974; $\mathrm{p} 257$.

24. Zhu, C.; Nakamura, H. J. Chem. Phys. 1994, 101, 4855. 